\title{
Switching the uniaxial magnetic anisotropy by ion irradiation induced compensation
}

\author{
Ye Yuan ${ }^{1,4 *}$, Teyri Amarouche ${ }^{2}$, Chi Xu ${ }^{1,4}$, Andrew Rushforth ${ }^{3}$, Roman Böttger ${ }^{1}$, Kevin Edmonds ${ }^{3}$, \\ Richard Campion $^{3}$, Bryan Gallagher ${ }^{3}$, Manfred Helm ${ }^{1,4}$, Hans Jürgen von Bardeleben ${ }^{2}$, and shengqiang Zhou ${ }^{1}$ \\ ${ }^{1}$ Helmholtz-Zentrum Dresden Rossendorf, Institute of Ion Beam Physics and Materials Research, Bautzner \\ Landstrasse 400, D-01328 Dresden, Germany \\ ${ }^{2}$ Institut des Nanosciences de Paris-INSP Université Pierre et Marie Curie and UMR 7588 au CNRS, Paris, \\ France \\ ${ }^{3}$ School of Physics and Astronomy, University of Nottingham, Nottingham NG7 2RD, United Kingdom \\ ${ }^{4}$ Technische Universität Dresden, D-01062 Dresden, Germany
}

\begin{abstract}
:
In the present work, the uniaxial magnetic anisotropy of GaMnAsP is modified by helium ion irradiation. According to the micro-magnetic parameters, e.g. resonance fields and anisotropy constants deduced from ferromagnetic resonance measurements, a rotation of the magnetic easy axis from out-of-plane [001] to in-plane [100] direction is achieved. From the application point of view, our work presents a novel avenue in modifying the uniaxial magnetic anisotropy in GaMnAsP with the possibility of lateral patterning by using lithography or focused ion beam.
\end{abstract}

*y.yuan@hzdr.de 
The controllable uniaxial magnetic anisotropy (MA) in dilute magnetic semiconductors (DMSs) has been intensely investigated due to its decisive functionality in spintronics [1,2]. The GaMnAs films grown on GaAs substrates generally exhibit a magnetic easy axis along the in-plane direction due to the compress strain [1]. To have perpendicular MA in GaMnAs, two approaches have been attempted. One is to grow tensile strained GaMnAs films on InGaAs buffer layers with a proper indium concentration [3], which can have a larger lattice constant than GaMnAs. Another approach is to tune the lattice constant of GaMnAs by co-alloying with phosphorus. The GaMnAsP compound can have a smaller lattice constant than the GaAs substrate by adjusting the $\mathrm{P}$ concentration such that the layers are under tensile strain and have an out-of-plane magnetic easy axis $[4,5]$. GaMnAsP films with out-of-plane MA provide an avenue for testing spintronic concepts in which a perpendicular magnetic easy axis is preferred $[4,6,7]$. According to the $p$ - $d$ Zener model, the hole density largely influences, in addition to the Curie temperature $\left(T_{\mathrm{C}}\right)$ and magnetization, the uniaxial MA through cooperating with strain-induced valence band splitting [8, 9]. One previous experimental confirmation was provided in hydrogenated compressively strained GaMnAs on GaAs substrates: The magnetic easy axis rotates from the in-plane to the out-of-plane direction, when the hole concentration is reduced by hydrogen compensation [10]. In addition, many experiments have been performed to seek various avenues to control MA through modifying the Fermi level, e.g. doping with sulfur-donors compensates holes in GaMnP, causing a MA reversal in the plane [11]. Contrary to hole compensation, low-temperature long-time annealing effectively increases the hole concentration and thereby shifts the Fermi level in the valence band down by removing interstitial $\mathrm{Mn}$ atoms in as-grown GaMnAsP samples. This leads to a switching of the the magnetic easy axis from the in-plane to the out-of-plane direction [12]. As mentioned above, exploring new ways to flexibly tune uniaxial MA [13] is still being recognized as a central task in DMSs.

One of the alternative approaches to tune the hole concentration in semiconductors is ion irradiation [14-17]. After irradiation, some deep-level trapping centers are generated in the irradiated region, compensating free carriers. As a result, the induced compensation modifies the magnetization, magnetic anisotropy and Curie temperature in DMSs [17]. Most importantly, according to previous studies, ion irradiation at low fluence presents a 
possibility of introducing defects meanwhile preserving the crystal structure, as verified by X-ray diffraction and Rutherford backscattering/channeling spectrometry $[17,18]$. However, in previous works $[14,17]$ the influence of the ion irradiation on the magnetic anisotropy, particularly the switch of magnetic easy axis between in- and out-of-plane directions, has not been discussed. Indeed, tuning magnetic anisotropy is technologically more important since ion irradiation combined with photolithography can provide lateral patterning on demand.

In the present work, we employ helium ion irradiation to tune the uniaxial magnetic anisotropy in GaMnAsP. The magnetic easy axis is changed from the out-of-plane to the in-plane direction upon increasing compensation, accompanied by a reduction of magnetization and Curie temperature. Ferromagnetic-resonance (FMR) measurements confirm the Zener model description: The second-order out-of-plane magnetic anisotropy constant is dominated by the hole concentration. In addition, the reduction of the itinerant hole concentration drives the metallic system across the insulator-metal transition into the insulating state.

All used samples were grown on semi-insulating (001) GaAs substrate at a low temperature of $230{ }^{\circ} \mathrm{C}$ by employing a Veeco Mod Gen III molecular beam epitaxy (MBE) system at the University of Nottingham [12]. The Mn $(x)$ and $\mathrm{P}$ concentration $(y)$ is 0.06 and 0.10 , respectively. Before growing the $25 \mathrm{~nm}$ GaMnAsP layer, a $50 \mathrm{~nm} \mathrm{GaAs}{ }_{0.9} \mathrm{P}_{0.1}$ buffer layer was pre-grown to reduce the lattice-mismatch on the GaAs substrate. All as-grown $\mathrm{Ga}_{0.94} \mathrm{Mn}_{0.06} \mathrm{As}_{0.90} \mathrm{P}_{0.10}$ samples were annealed in air at $190{ }^{\circ} \mathrm{C}$ for 48 hours to out-diffuse $\mathrm{Mn}$ interstitials.

The helium-ion irradiation was done at room temperature at the Ion Beam Center (IBC) of Helmholtz-Zentrum Dresden-Rossendorf (HZDR). The irradiation energy was set as $4 \mathrm{keV}$. The displacement per atom (DPA) was calculated by using the Stopping and Range of Ions in Matter (SRIM) code [19]. Different from ion implantation process, we used helium noble gas ions which will not introduce any doping effect but only defects along their penetration paths. According to the SRIM simulation shown in Fig. 1, the peak of DPA locates at a depth of around $15 \mathrm{~nm}$, producing a relatively uniform defect distribution in the top $25 \mathrm{~nm}$ GaMnAsP. However, the implanted helium ions mainly stay as a Gaussian distribution with a peak at around $36 \mathrm{~nm}$ below the 
surface, i.e. below the top GaMnAsP layer. The helium-ion fluences were chosen as $1 \times 10^{13}, 2 \times 10^{13}$, and $3 \times 10^{13} \mathrm{~cm}^{-2}$ for samples $1 \mathrm{E} 13,2 \mathrm{E} 13$, and $3 \mathrm{E} 13$, respectively, as shown in Table I. Magnetic field and temperature dependent magnetization curves were measured by a superconducting quantum interference device (Quantum Design, SQUID-VSM) magnetometer. The temperature dependent remnant magnetization of all samples was measured under zero-field during warming process, which was after cooling from $300 \mathrm{~K}$ down to $5 \mathrm{~K}$ under a $5 \mathrm{kOe}$ field. For all samples, the diamagnetic signal at $5 \mathrm{~K}$ was subtracted by the susceptibility measured from the $\mathrm{MH}$ curve at room temperature where the diamagnetism from substrate dominates. Electric transport properties were measured using a commercial Lake Shore Hall Measurement System in the van der Pauw geometry.

TABLE I. Irradiation fluences, peak DPA, Curie temperatures, and the out-of-plane second order anisotropy constants of referenced and irradiated GaMnAsP samples. The sample 1E13 is superparamagnetic, so only a blocking temperature $\left(T_{\mathrm{B}}\right)$ is determined.

\begin{tabular}{ccccc}
\hline \hline Sample No. & Implantation fluences & Peak DPA & $T_{\mathrm{C}}$ & $K_{2 \perp}(5 \mathrm{~K})$ \\
\hline Virgin & Virgin & 0 & $76 \mathrm{~K}$ & $22.1 \times 10^{3} \mathrm{erg} / \mathrm{cm}^{3}$ \\
$1 \mathrm{E} 13$ & $1 \times 10^{13} \mathrm{~cm}^{-2}$ & $0.64 \times 10^{-3}$ & $53 \mathrm{~K}$ & $5.6 \times 10^{3} \mathrm{erg} / \mathrm{cm}^{3}$ \\
$2 \mathrm{E} 13$ & $2 \times 10^{13} \mathrm{~cm}^{-2}$ & $1.28 \times 10^{-3}$ & $38 \mathrm{~K}$ & $-18.3 \times 10^{3} \mathrm{erg} / \mathrm{cm}^{3}$ \\
$3 \mathrm{E} 13$ & $3 \times 10^{13} \mathrm{~cm}^{-2}$ & $1.92 \times 10^{-3}$ & $22 \mathrm{~K}\left(T_{\mathrm{B}}\right)$ & - \\
\hline \hline
\end{tabular}

The temperature dependent sheet resistivities of all samples are shown in Fig. 2. For the unirradiated, virgin sample, the sheet resistivity exhibits a moderate increase upon lowering the temperature down to $97 \mathrm{~K}$, below which the resistivity slightly decreases. Moreover, a positive value of $d \rho / d T$ is obtained at the lowest reached temperature. Upon increasing the irradiation fluence, the resistivity gradually increases by more than three orders of magnitude due to hole compensation, rendering the samples insulating. This is particularly for the sample 3E13 with the highest irradiation dose, which shows a nearly constant hopping energy when plotted versus $T^{1 / 4}$ (Fig. 2b). A direct measurement of hole concentrations in GaMnAs by Hall effect is challenging due to the appearance of anomalous Hall effect [20]. The reduction of hole concentration in ion irradiated GaMnAs has been confirmed in refs.[14] and [17]. In ref. 14, 
the authors have measured the decreased hole concentration in GaAs:Zn after $\mathrm{Ne}$ irradiation by electrochemical capacitance voltage (ECV) profiling to calculate the carrier removal rate. And the deduced linear hole-compensation rate has been used to calculate the hole concentration in GaMnAs.

The irradiation-tuned magnetization of three samples is shown in Fig. 3. After subtracting the diamagnetic background from substrates, highly square-like hysteresis loops (shown in insets of Figs. 3a, b, and c) together with concave mean-field-like remnant magnetization curves (shown in Figs. 3a, b and c) confirm the ferromagnetic coupling in these three samples (Virgin, 1E13, and 2E13). Upon increasing irradiation fluence, a decreased $T_{\mathrm{C}}$ is observed. Such a result is consistent with the $p-d$ Zener model prediction [17]: $T_{\mathrm{C}} \sim x p^{1 / 3}$, where $x$ is the effective substitutional Mn concentration and $p$ is the hole concentration. The irradiation induced defects compensate holes in the layer, leading to a reduction of $T_{\mathrm{C}}$. As displayed in Fig. $3 \mathrm{~d}$, the $T_{\mathrm{C}}$ reduces linearly upon increasing He ion fluences. In addition to magnetization and $T_{\mathrm{C}}$, the uniaxial magnetic anisotropy is also tuned. The magnetic easy axis gradually shifts from the outof-plane to the in-plane direction upon increasing DPA. For the reference sample Virgin, an out-of-plane magnetic anisotropy appears, which is confirmed by the highly square-like hysteresis loop and the concave mean-field-like remanence curve when the magnetic field is applied along the out-of-plane direction. Such a perpendicular magnetic anisotropy results from the tensile strain induced valence band splitting when phosphorus atoms substitute arsenic sites [4]. In sample 2E13, a magnetic anisotropy reset is verified by exchanging line shapes of $M H$ curves when the field is along out-of-plane and in-plane directions. When the magnetic field is applied along the out-of-plane direction, multiple switching events occur (see the open loop in MH curve in the inset to Fig. 3c). Such multiple switching events were also observed in as-grown (by LT-MBE) GaMnAsP samples [12]. This is due to the competition between strain-induced magnetic anisotropy, cubic magneto-crystalline anisotropy and shape anisotropy [12]. It is worth noting that sample 1E13 performs a transition feature according to the open hysteresis loops and similar remnant magnetization for both directions at $5 \mathrm{~K}$ in Fig. 3c. The MA switching can be explained by the fact that the $\mathrm{P}$ doping induced tensile-strain in the GaMnAsP layer causes a splitting of heavy-hole/light-hole bands of GaAs at $k=0$ [9]. 
When the hole concentration is changed, the Fermi level moves between different subbands. This has been predicted in both compressively and tensily strained GaAs [9]. In compressively strained GaMnAs, Sawicki et al. has observed that the magnetic easy axis is switched from in-plane [100] to out-ofplane [001] when the hole concentration is decreased [21]. Compared to the compressively strained GaMnAs, in our case the GaMnAsP films are under tensile strain. The hole concentrations are decreased due to ion irradiation and the Fermi-level moves up. The magnetic easy axis is switched from out-of-plane [001] to in-plane [100].

Ferromagnetic resonance spectra were measured with an X-band $(9.4 \mathrm{Ghz})$ spectrometer. To improve the signal / noise of the experiment, the FMR measurement was performed using a magnetic field modulated at $100 \mathrm{kHz}$. Therefore, the measured FMR signal is proportional to the first derivative. For all measurements, two configurations are used to describe the ferromagnetic resonance behavior: The polar and azimuthal angles of applied field $H\left(\theta_{\mathrm{H}}, \varphi_{\mathrm{H}}\right)$, and the equilibrium magnetization $M(\theta, \varphi)$, are described in Fig. 4a. In order to explain the FMR results, the Smit-Beljers approach [22] is employed to obtain the free energy density $F$ :

$$
\begin{gathered}
F=-M H\left[\cos \theta \cos \theta_{H}+\sin \theta \sin \theta_{H} \cos \left(\varphi-\varphi_{H}\right)\right]-2 \pi M^{2} \sin ^{2} \theta- \\
K_{2 \perp} \cos ^{2} \theta-\frac{1}{2} K_{4 \perp} \cos ^{4} \theta-\frac{1}{2} K_{4} \frac{(3+\cos 4 \varphi)}{4} \sin ^{4} \theta-K_{2} \sin ^{2} \theta \sin ^{2}\left(\varphi-\frac{\pi}{4}\right) \\
\left(\frac{\omega}{\gamma}\right)^{2}=\frac{1}{M_{S} \sin ^{2} \theta}\left[\frac{\partial^{2} F}{\partial \theta^{2}} \frac{\partial^{2} F}{\partial \varphi^{2}}-\left(\frac{\partial^{2} F}{\partial \theta \partial \varphi}\right)^{2}\right]
\end{gathered}
$$

where the first term is the Zeeman energy; the second term is the shape anisotropy (related to the demagnetization energy); $K_{2 \perp}$ represents the perpendicular uniaxial anisotropy which is induced by biaxial strain; $K_{4}$ and $K_{4 \perp}$ are the biaxial strain modified cubic magneto-crystalline anisotropy constants which are related to the GaAs zinc-blende crystal structure; $K_{2}$ is a uniaxial term which is related to an additional anisotropy between [110] and [1 $\overline{1} 0]$ directions [23]; According to the Smit-Beljers equation [24], one can obtain the FMR conditions for an arbitrary magnetic field direction as shown in Eq. (2): $\omega$ is the angular frequency of the microwave field, and $\gamma$ is the gyromagnetic ratio. By 
minimizing the free energy $F$ with respect to $\theta$ and $\varphi$ in Eq. (1) [25], one can obtain anisotropy constants $K_{2 \perp}, K_{2}, K_{4 \perp}$, and $K_{4}$, respectively.

Figures $4 \mathrm{~b}, \mathrm{c}$ and $\mathrm{d}$ show the angular dependence of resonance fields of samples Virgin and 1E13. For all samples, the dc magnetic field was varied in the (001), (110), (010), and (1-10) planes, and the lowest resonance field exhibits in the magnetic easy axis direction. As shown in Fig. 4b, at temperatures of $5,10,20,40$, and $60 \mathrm{~K}$, the [001] (out-of-plane) direction expresses a much easier magnetic behavior than the [110] direction. Moreover, according to the temperature dependent resonance field plots in Fig. 5a, magnetic moments prefer to lie along the out-of-plane direction than any other axis in the plane in the whole temperature range, which is a typical feature in a low compensated tensile strained III-V DMS layer, eg. InMnAs films on InAs substrates [26, 27] and GaMnAs films on InGaAs substrates [28]. Interestingly, in the (001) plane the [100] direction acts as a much easier axis than the [110] and the $[1 \overline{1} 0]$ directions. Such [100] oriented cubic anisotropy comes from the spin anisotropy of the hole liquid, originating from a strong spin-orbit coupling in the host material's valence band, thus depends on the epitaxial strain, hole concentration, and temperature [9]. It is worth noting that the resonance fields

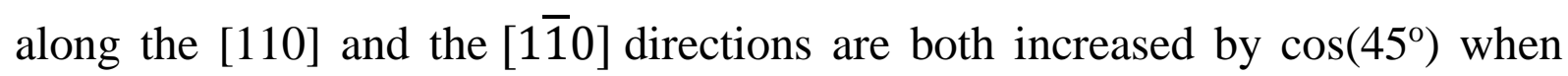
compared with the one along the [100] direction, indicating that the cubic anisotropy dominates in the (001) plane. This can be further confirmed by the comparable resonance fields along the [110] and the [1 $\overline{1} 0]$ directions. It is worth noting that the in-plane uniaxial magnetic anisotropy which usually happens in low-compensated GaMnAs is also present, particularly in the high temperature range, in the studied films, probably coming from the Mn-Mn dimer construction [23]. For sample 1E13, irradiation induced compensation starts to rotate the magnetic easy axis to the (001) plane. As displayed in Fig. 4c, when the magnetic field rotates from [110] to [001], a maximal value appears when the angle approaches 45 degree, resulting from a competition between the inplane cubic anisotropy and the tensile strain induced uniaxial magnetic anisotropy along the out-of-plane direction. In the (001) plane, the magnetic easy axis still stays along the [100] direction rather than along [110] and [1 $\overline{1} 0]$ directions. However, according to Fig. 5b, comparable resonance fields along 
[001] and [100] directions indicate an intermediate state of the easy axis rotation. Upon increasing irradiation dose to $2 \times 10^{13} / \mathrm{cm}^{2}$, the magnetic easy axis is totally along the [100] direction in sample 2E13. Interestingly, in these three samples (Virgin, 1E13 and 2E13) the magnetic easy axis in the (001) plane is always along the [100] direction.

To obtain temperature dependent anisotropy constants from FMR measurements, Eq. 1 is employed, and the anisotropy fields are calculated by $\mathrm{H}$ $=2 K_{i} / \mathrm{M}$. For the reference sample Virgin, the second order anisotropy constant $K_{2 \perp}$ is around $1 \mathrm{kOe}$ at $5 \mathrm{~K}$ and presents much larger than the other anisotropy constants till $60 \mathrm{~K}$, confirming an out-of-plane magnetic easy axis. However, upon increasing the irradiation fluence, $K_{2 \perp}$ gradually turns negative, reflecting the suppression of $K_{2 \perp}$ by decreasing hole concentration. It is worth noting that $K_{2 \perp}$ decreases monotonically with raising the irradiation fluence as depicted in Fig. 6d, which exhibits a same trend like $T_{\mathrm{C}}$ vs. fluence as presented in Fig. $3 \mathrm{~d}$. According to the mean field theory, $K_{2 \perp}$ should vary linearly upon changing hole concentration [29]. In sample 1E13, the cubic anisotropy constant $K_{4}$ is enhanced, gradually overcoming the decreased $K_{2 \perp}$ constant. Such competition directly results in an enhancement of the resonance field along the [111] direction, as shown in Fig. 4c. In the highly compensated sample 2E13, the uniaxial magnetic anisotropy constants $K_{2}$ and $K_{2 \perp}$ are largely suppressed, while two cubic anisotropy constants, $K_{4}$ and $K_{4 \perp}$ dominate.

Note that the sample 3E13 is overcompensated by $\mathrm{He}^{+}$irradiation, only a superparamagnetic behavior is observed [17], thus no anisotropic FMR signal is expected.

In summary, the magnetic field and temperature dependent magnetization as well as the micro-magnetic parameters prove that the magnetic easy axis rotates from the out-of-plane to the in-plane direction with decreasing hole concentration by ion irradiation in GaMnAsP films. This observation is in agreement with the Zener model prediction for the tensile strained III-Mn-V DMSs. Ion irradiation together with proper lithography can easily realize lateral patterning and meet the requirement for spintronic device fabrications. 


\section{ACKNOWLEDGMENTS}

Support by the Ion Beam Center (IBC) at HZDR is gratefully acknowledged.

This work is funded by the Helmholtz-Gemeinschaft Deutscher

Forschungszentren (HGF-VH-NG-713). The author Y. Y. thanks financial support by Chinese Scholarship Council (File No. 201306120027).

[1] Dietl T and Ohno H 2014 Dilute ferromagnetic semiconductors: Physics and spintronic structures Rev. Mod. Phys. 86187

[2] Dietl T 2010 A ten-year perspective on dilute magnetic semiconductors and oxides Nat. Mater. 9 965-74

[3] Glunk M, Daeubler J, Dreher L, Schwaiger S, Schoch W, Sauer R, Limmer W, Brandlmaier A, Goennenwein S T B, Bihler C and Brandt M S 2009 Magnetic anisotropy in (Ga,Mn)As: Influence of epitaxial strain and hole concentration Phys Rev B 79195206

[4]Cubukcu M, von Bardeleben H J, Khazen K, Cantin J L, Mauguin O, Largeau L and Lemaitre A 2010 Adjustable anisotropy in ferromagnetic (Ga,Mn)(As,P) layered alloys Phys. Rev. B $\mathbf{8 1}$ 041202(R)

[5] Yahyaoui M, Boujdaria K, Cubukcu M, Testelin C and Gourdon C 2013 The influence of phosphorus content on magnetic anisotropy in ferromagnetic $(\mathrm{Ga}, \mathrm{Mn})(\mathrm{As}, \mathrm{P}) / \mathrm{GaAs}$ thin films J. Phys.: Condens. Matter 25346001

[6] Thevenard L, Gourdon C, Haghgoo S, Adam J P, von Bardeleben H J, Lemaitre A, Schoch W and Thiaville A 2011 Domain wall propagation in ferromagnetic semiconductors: Beyond the one-dimensional model Phys. Rev. B 83245211

[7] Gorchon J, Curiale J, Cebers A, Lemaitre A, Vernier N, Plapp M and Jeudy V 2015 Current-induced fingering instability in magnetic domain walls Phys. Rev. B 92 060411(R)

[8] Dietl T, Ohno H, Matsukura F, Cibert J and Ferrand D 2000 Zener model description of ferromagnetism in zinc-blende magnetic semiconductors Science 287 1019-22

[9] Dietl T, Ohno H and Matsukura F 2001 Hole-mediated ferromagnetism in tetrahedrally coordinated semiconductors Phys. Rev. B 63195205

[10] Khazen K, von Bardeleben H J, Cantin J L, Thevenard L, Largeau L, Mauguin O and Lemaitre A 2008 Ferromagnetic resonance of $\mathrm{Ga}_{0.93} \mathrm{Mn}_{0.07} \mathrm{As}$ thin films with constant $\mathrm{Mn}$ and variable free-hole concentrations Phys. Rev. B 77165204

[11] Stone P R, Bihler C, Kraus M, Scarpulla M A, Beeman J W, Yu K M, Brandt M S and Dubon O D 2008 Compensation-dependent in-plane magnetization reversal processes in $\mathrm{Ga}_{1-x} \mathrm{Mn}_{x} \mathrm{P}_{1-y} \mathrm{~S}_{y}$ Phys. Rev. B $\mathbf{7 8} 214421$

[12] Casiraghi A, Rushforth A W, Wang M, Farley N R S, Wadley P, Hall J L, Staddon C R, Edmonds K W, Campion R P, Foxon C T and Gallagher B L 2010 Tuning perpendicular magnetic anisotropy in $(\mathrm{Ga}, \mathrm{Mn})(\mathrm{As}, \mathrm{P})$ by thermal annealing Appl. Phys. Lett. 97122504

[13] Chiba D, Sawicki M, Nishitani Y, Nakatani Y, Matsukura F and Ohno H 2008 Magnetization vector manipulation by electric fields Nature 455 515-8

[14] Mayer M A, Stone P R, Miller N, Smith H M, Dubon O D, Haller E E, Yu K M, Walukiewicz W, Liu X and Furdyna J K 2010 Electronic structure of $\mathrm{Ga}_{1-x} \mathrm{Mn}_{x}$ As analyzed according to hole-concentration-dependent measurements Phys. Rev. B 81045205

[15] Sinnecker E H C P, Penello G M, Rappoport T G, Sant'Anna M M, Souza D E R, Pires M P, Furdyna J K and Liu X 2010 Ion-beam modification of the magnetic properties of $\mathrm{Ga}_{1-x} \mathrm{Mn}_{x}$ As epilayers Phys. Rev. B 81245203

[16] Li L, Yao S D, Zhou S Q, Burger D, Roshchupkina O, Akhmadaliev S, Rushforth A W, Campion R P, Fassbender J, Helm M, Gallagher B L, Timm C and Schmidt H 2011 Tailoring the magnetism of GaMnAs films by ion irradiation J. Phys. D: Appl. Phys. 44 045001 
[17] Zhou S Q, Li L, Yuan Y, Rushforth A W, Chen L, Wang Y T, Bottger R, Heller R, Zhao J H, Edmonds K W, Campion R P, Gallagher B L, Timm C and Helm M 2016 Precise tuning of the Curie temperature of $(\mathrm{Ga}, \mathrm{Mn})$ As-based magnetic semiconductors by hole compensation: Support for valence-band ferromagnetism Phys. Rev. B 94075205

[18] Li L, Yao S D, Roshchupkina O, Prucnal S, Akhmadaliev S, Campion R P, Rushforth A W, Fassbender J, Helm M, Gallagher B L, Timm C, Schmidt H and Zhou S Q 2011 Characterization and local magnetic modification of ion irradiated GaMnAs Nucl. Instrum. Meth. B $2692469-73$

[19] Ziegler J F 2004 Srim-2003 Nucl. Instrum. Meth. B 219 1027-36

[20] Omiya T, Matsukura F, Dietl T, Ohno Y, Sakon T, Motokawa M and Ohno H 2000 Magnetotransport properties of $(\mathrm{Ga}, \mathrm{Mn})$ As investigated at low temperature and high magnetic field Physica E 7 976-80

[21] Sawicki M, Matsukura F, Idziaszek A, Dietl T, Schott G M, Ruester C, Gould C, Karczewski G, Schmidt G and Molenkamp L W 2004 Temperature dependent magnetic anisotropy in (Ga,Mn)As layers Phys. Rev. B 70245325

[22] Smit J B, H. G. 1955 Philips Res. Rep. 10113

[23] Birowska M, Sliwa C, Majewski J A and Dietl T 2012 Origin of Bulk Uniaxial Anisotropy in Zinc-Blende Dilute Magnetic Semiconductors Phys. Rev. Lett. 108237203

[24] Titova L V, Kutrowski M, Liu X, Chakarvorty R, Lim W L, Wojtowicz T, Furdyna J K and Dobrowolska M 2005 Competition between cubic and uniaxial anisotropy in $\mathrm{Ga}_{1-}$ ${ }_{x} \mathrm{Mn}_{x} \mathrm{As}$ in the low-Mn-concentration limit Phys. Rev. B 72165205

[25] Farle M 1998 Ferromagnetic resonance of ultrathin metallic layers Rep. Prog. Phys. 61 $755-826$

[26] Zhou S Q, Wang Y T, Jiang Z N, Weschke E and Helm M 2012 Ferromagnetic InMnAs on InAs Prepared by Ion Implantation and Pulsed Laser Annealing Appl. Phys. Express 5 093007

[27] Yuan Y, Wang Y, Gao K, Khalid M, Wu C, Zhang W, Munnik F, Weschke E, Baehtz C, Skorupa W, Helm M and Zhou S 2015 High Curie temperature and perpendicular magnetic anisotropy in homoepitaxial InMnAs films J. Phys. D: Appl. Phys. 48235002

[28] Gourdon C, Dourlat A, Jeudy V, Khazen K, von Bardeleben H J, Thevenard L and Lemaitre A 2007 Determination of the micromagnetic parameters in (Ga,Mn)As using domain theory Phys. Rev. B 76241301

[29] Abolfath M, Jungwirth T, Brum J and MacDonald A H 2001 Theory of magnetic anisotropy in $\mathrm{III}_{1-x} \mathrm{Mn}_{x} \mathrm{~V}$ ferromagnets Phys. Rev. B 63054418 


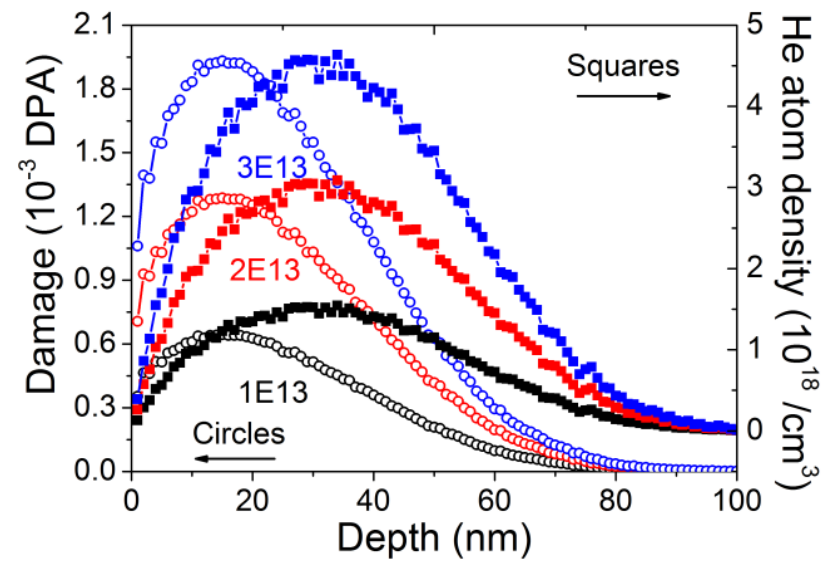

FIG. 1 (Color online) SRIM calculated damage profiles (DPA) (circles) and helium ion distribution (squares) profiles for samples 1E13 (black), 2E13 (red), and 3E13 (blue). 

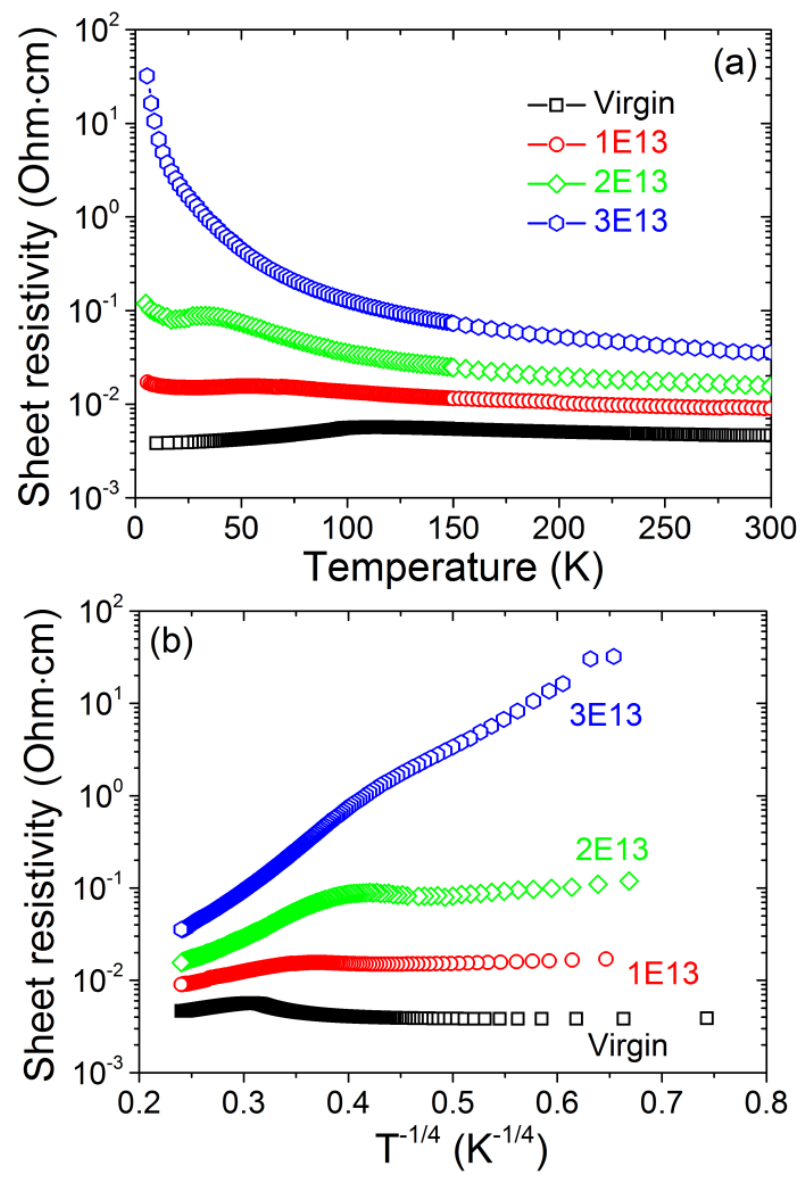

FIG. 2 (Color online) (a) Temperature and (b) temperature ${ }^{-1 / 4}$ dependent sheet resistivity of reference Virgin (circles) and irradiated 1E13 (squares), 2E13 (diamonds), and 3E13 (hexagons) samples. 

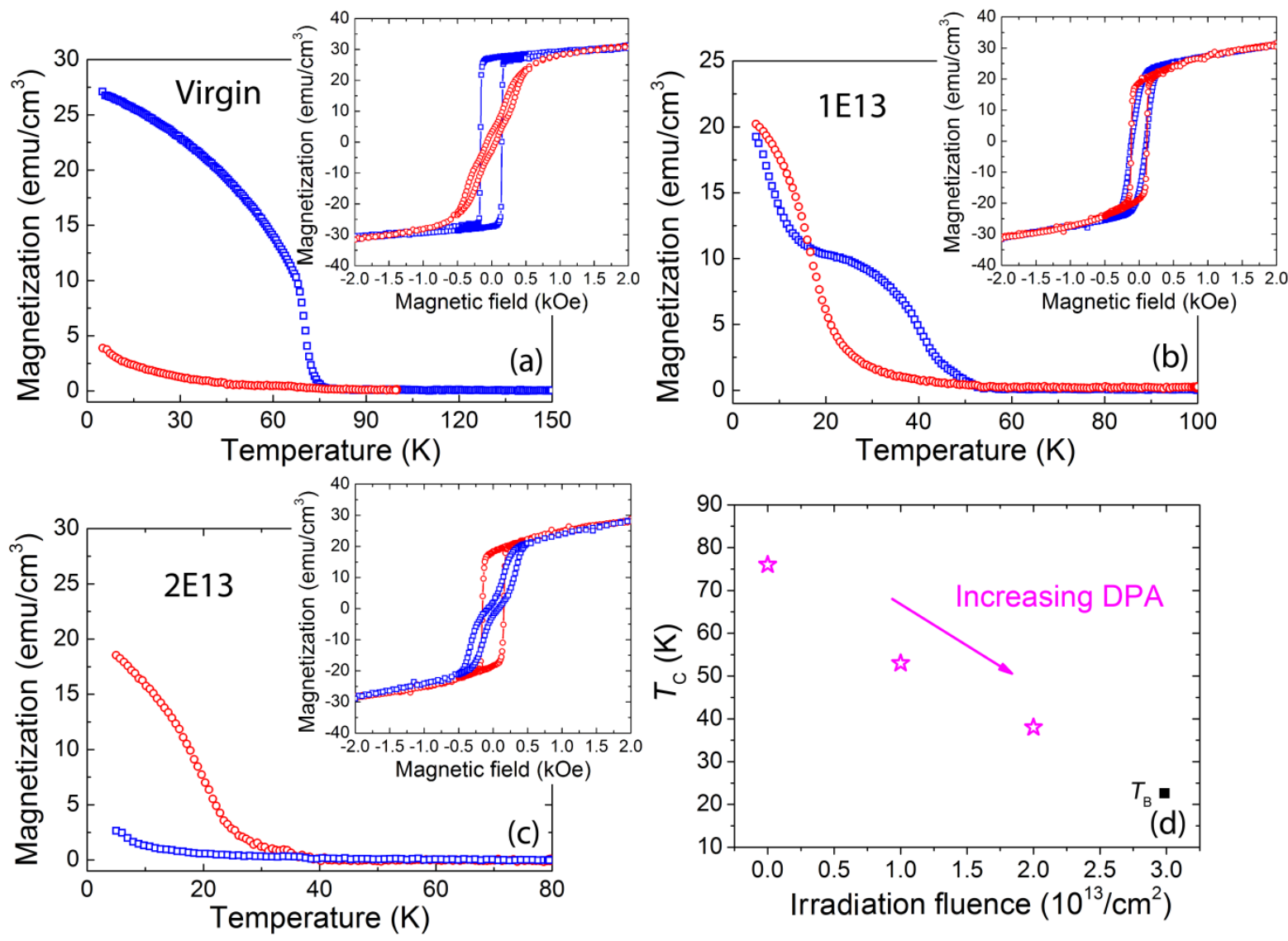

FIG. 3 (Color online) (a), (b), (c) Temperature dependent remnant magnetization under zerofield of samples (a) Virgin, (b) 1E13, and (c) 2E13 when magnetic field is applied along outof-plane (squares) and in-plane directions (circles). Insets show the magnetic field dependent magnetization at $5 \mathrm{~K}$ when magnetic field is along out-of-plane (squares) and in-plane [100] directions (circles). (d) Irradiation fluence dependent Curie temperatures (stars) and blocking temperature (square) of all samples. 
(a)
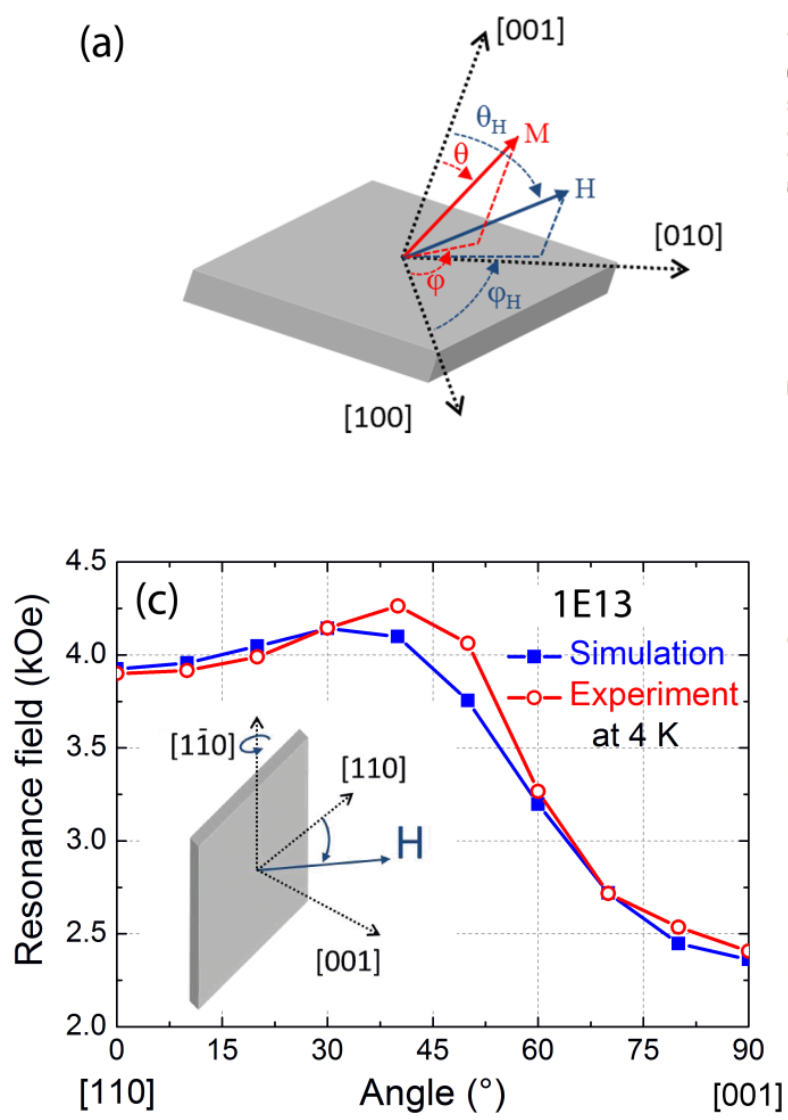
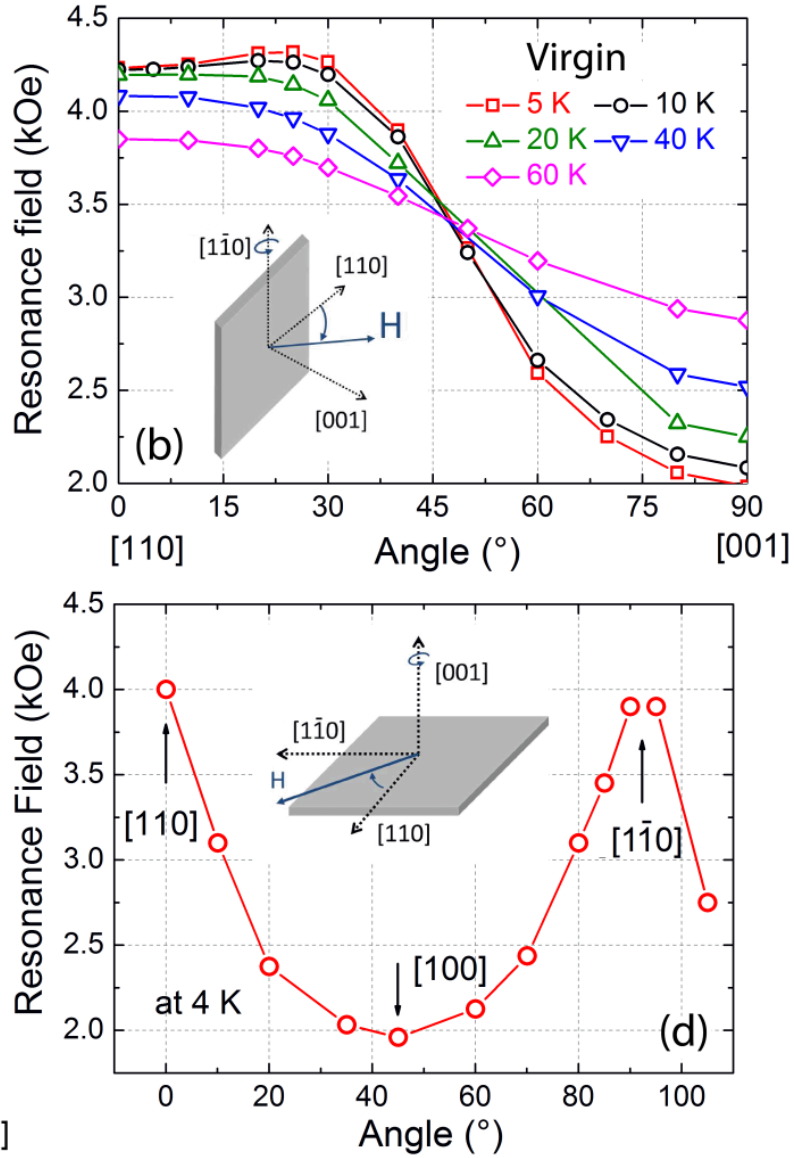

FIG.4 (Color online) (a) Coordinate system used in this paper. The magnetic field direction can be rotated continuously through the polar and azimuthal angles (depicted as $\theta_{\mathrm{H}}$ and $\varphi_{\mathrm{H}}$ ). The magnetization is described by $(\theta, \varphi)$. Angular dependent FMR resonance fields for samples (b) Virgin at 5,10, 20, 40, and $60 \mathrm{~K}$ and (c) (d) $1 \mathrm{E} 13$ at $4 \mathrm{~K}$ with two different configurations. The configurations are given as insets in the figure. 

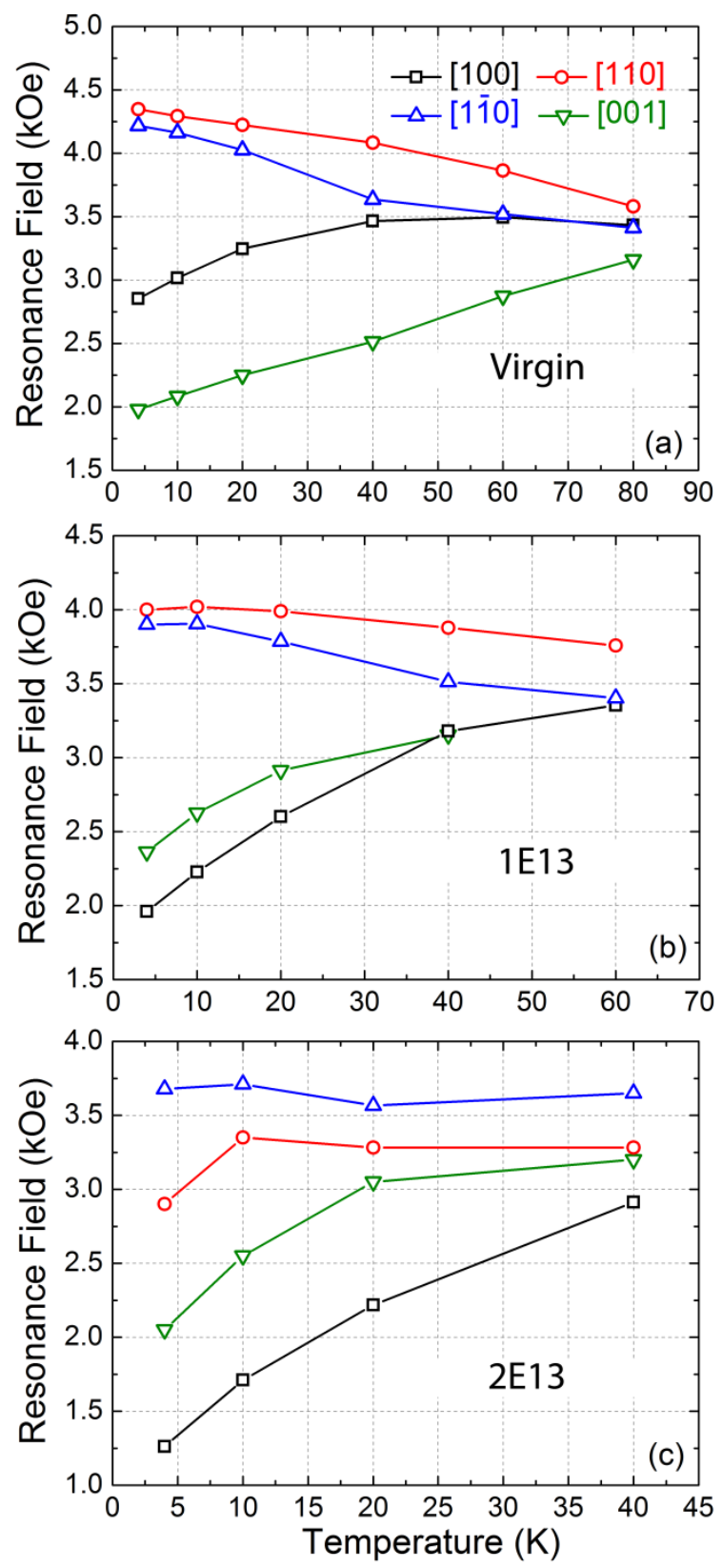

FIG. 5 (Color online) Temperature dependent resonance fields when the dc magnetic field is along [100] (squares), [001] (down triangles), [110] (circles), and [1̄ㅣ] (up triangles) for samples (a) Virgin (b) 1E13, and (c) 2E13. 

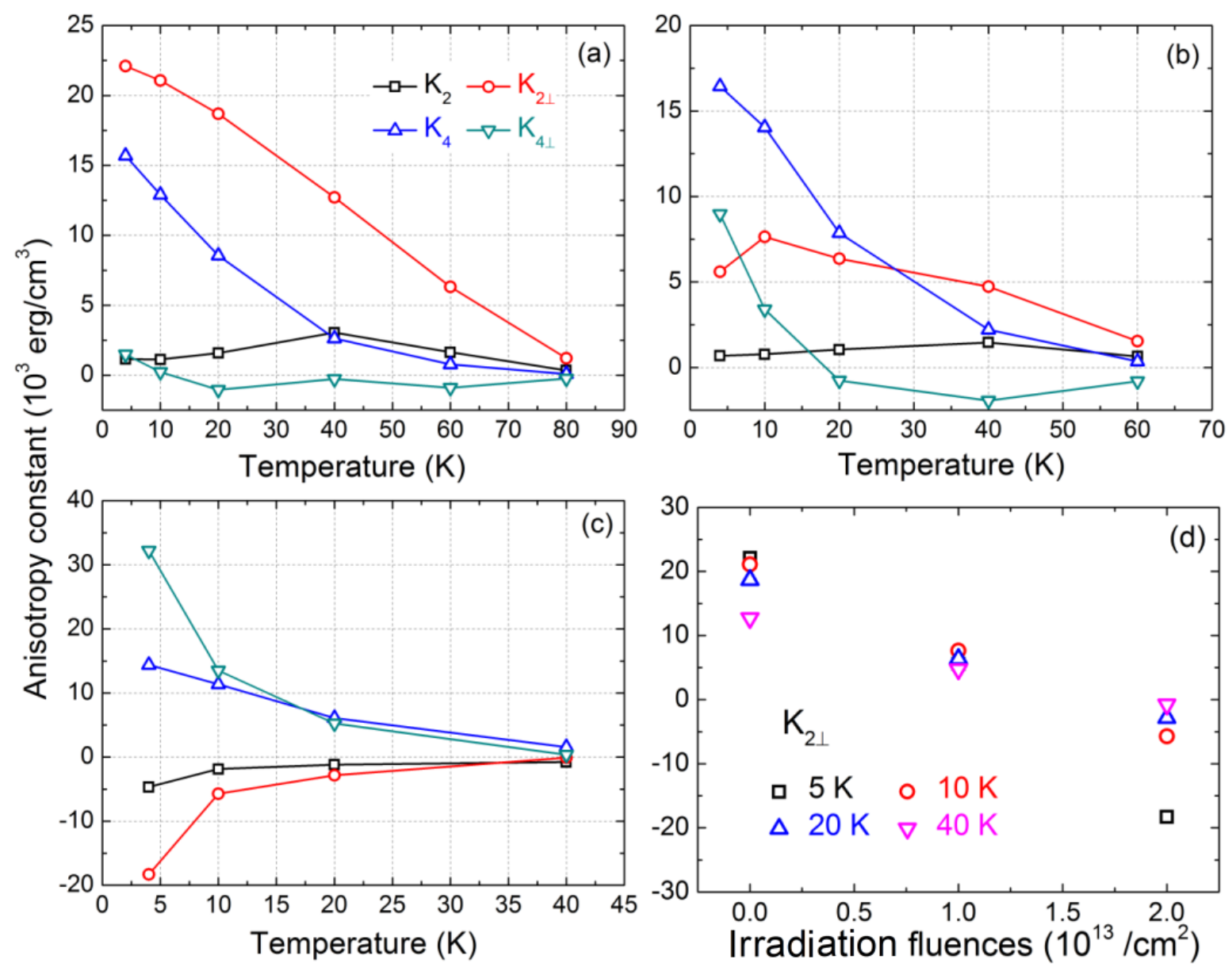

FIG. 6 (Color online) Temperature dependence of anisotropy parameters obtained from the ferromagnetic resonance of samples (a) Virgin, (b) 1E13, and (c) 2E13. (d) Irradiation fluence dependent second order perpendicular uniaxial magnetic anisotropy constants at 5, 10, 20, and $40 \mathrm{~K}$. 